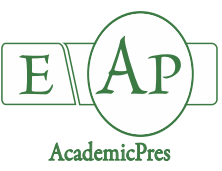

Morales-Manzo I-I et al. (2021)

Notulae Botanicae Horti Agrobotanici Cluj-Napoca

Volume 49, Issue 2, Article number 12354

DOI: $10.15835 /$ nbha49212354

Research Article

\title{
Novel sources of resistance to powdery mildew (Leveillula taurica (Lév.) Arnaud) in pepper
}

\section{Ivan-Ilich MORALES-MANZOํㅜ, Adrián RODRÍGUEZ-BURRUEZO', Marisa JIMÉNEZ-PÉREZ1 ${ }^{1}$, José J. LUNA-RUIZ², Alberto SAN-BAUTISTA ${ }^{3}$, Ana FITA ${ }^{1 *}$}

\author{
${ }^{1}$ Universitat Politècnica de València, Instituto de Conservación y Mejora de la Agrodiversidad Valenciana (COMAV), Camino de \\ Veras/n,46022,Valencia,Spain; ivmoman@posgrado.upv.es; adrodbur@upvnet.upv.es; majipe1@posgrado.upv.es; \\ anfifer@btc.upv.es ('corresponding author) \\ ${ }^{2}$ Universidad Autónoma de Aguascalientes (UAA), Centro de Ciencias Agropecuarias, Avenida Universidad 940, Aguascalientes, \\ Aguascalientes 20100, Mexico; joselunaruiz11@yahoo.com.mx \\ ${ }^{3}$ Universitat Politècnica de València, Centro Valenciano de Estudios sobre Riego - Dep. Producción Vegetal, Camino de Vera s/n, \\ 46022,Valencia,Spain; asanbau@prv.upv.es
}

\begin{abstract}
Peppers, a worldwide crop, are threatened by different pathogens. Powdery mildew, a biotroph fungal infection, can cause several damages directly on vegetative parts and indirectly on fruits. Despite some sources of resistance have been described, commercial genotypes only with partial resistance have been developed due to the complex nature of such resistance and variable genetic expression, which depends on the stage of the plants. In this paper 49 accessions from different Capsicum species and origins have been tested. Plants were grown in growth chambers inside of mini greenhouses. Repeated inoculations under pepper leaves were applied by spraying a suspension of $10^{4}$ conidia $\mathrm{ml}^{-1}$. Readings were made at 30 and 60 days after inoculation (DAI). Total number of leaves (TL), total number of affected leaves (LA), and maximum area affected (MAA) in the most damaged leaf were scored. In addition, a composite infection index (CII) was calculated on the basis of the three mentioned traits. Inoculated plants showed more severe symptoms at 30 DAI than at 60 DAI. Different response patterns were observed: from accessions suffering high leaf shedding to some others with local hypersensitive response, indicating different gene action. The use of CII prevented species bias and disease response. In the present work, four highly tolerant accessions were identified, including two chiltepins, $C$. annuum wild relatives, Ag-01 and Ag-02, and two C. annuum A-06 and A-23.
\end{abstract}

Keywords: chiltepin; high tolerance; oidium; chlorosis; complex control resistance; screening

\section{Introduction}

Peppers (Capsicum spp.) are one of the most important horticultural crops grown worldwide and adapted to a range of growing conditions, which are used as both fresh or processed (FAOSTAT, 2021). As a widespread crop it is threaten by different pathogens. Powdery mildew (Leveillula taurica (Lév.) Arn.) is one of them, mainly in cool and humid environments, as greenhouses and open fields in mid-latitudes during autumn

Received: 26 Apr 2021. Received in revised form: 03 Jun 2021. Accepted: 04 Jun 2021. Published online: 08 Jun 2021.

From Volume 49, Issue 1, 2021, Notulae Botanicae Horti Agrobotanici Cluj-Napoca journal uses article numbers in place of the traditional method of continuous pagination through the volume. The journal will continue to appear quarterly, as before, with four annual numbers. 
and winter seasons (Parisi et al., 2020). Infection of this obligate fungal endoparasite biotroph starts when airborne conidia (asexual spores) lands over upper leaf surface, germinates and hypha enters by stomata or any other aperture, developing his mycelium inside mesophyll leaves (Zheng et al., 2013). First symptoms are yellow chlorotic spots in the oldest leaves. Later, the development of conidiophores on abaxial leaves (underside) provokes the characteristic white powder of this disease. Premature leaf shedding (or leaf abscission) is induced, affecting production by both decreasing photosynthesis and exposing fruits to sunscald (Molot et al., 1990).

The biotroph nature of the fungus requires to maintain infected plants as a source of inoculum and limits the evaluation of accessions for resistance (Daubeze et al., 1995; Lefebvre et al., 2003; Blat et al., 2005 and 2006). Evaluation usually is done in open fields at the fruiting stage, but it also can be done under controlled conditions (greenhouses or growth chambers) during early plant stages, where inoculation of accessions is done from 3-4 true leaves up to the fruiting stage (de Souza and Café-Filho, 2003; Zheng et al., 2013). The response in early stages can differ from those in adult plants. For instance, some $C$. annuum L. commercial accessions have showed better responses against the infection in young plants (de Souza and Café-Filho, 2003). Three main inoculation techniques are commonly used: i) natural infection, ii) artificial inoculation by directly brushing conidia on leaves; iii) spraying conidia suspensions of variable concentration $\left(10^{5}-2.5 \times 10^{4} \mathrm{ml}^{-1}\right)$ with Triton X $1001 \mu \mathrm{l} \mathrm{ml} l^{-1}$ (Molot et al., 1990; Daubeze et al., 1995; Zheng et al., 2013; Albert et al., 2017; Özer et al., 2018; McCoy and Bosland, 2019). Controlled screenings have been performed using a wide range of temperatures (16-27 ${ }^{\circ} \mathrm{C}$, Molot et al., 1987; Zheng et al., 2013), humidity (30-70\% $\pm 15 \mathrm{RH}$, Bai et al., 2003; Zheng et al., 2012) and photoperiod (12-14 h light and 12-10 h dark, Molot et al., 1987; Özer et al., 2018). To evaluate properly the response to infection some authors have suggested semi-quantitative scoring system or variants (Molot et al., 1987). Such methodology ranks the plants using a scale from 0 to 5 , where $0=$ to healthy plant (i. e., no infested leaves) and $5=80-100 \%$ plant foliage infested. Daubeze et al. (1995) used a similar scoring system called sporulation intensity ( $\mathrm{Sp}, 0-5$ scale), but also evaluated the proportion of diseased leaf area per plant (Pr, 0-5 scale) using a semi-quantitative scale too.

Although some sources of tolerance to powdery mildew (PM) have been reported in peppers, no complete resistance has been found. One of the first and most resistant $C$. annuum accessions identified were $\mathrm{H} 3$ (a pungent small-fruited Ethiopian powdery mildew resistant accession) and HV-12, an accession obtained as haplodiploid from hybrids of H3 and cv. 'Vania' (Daubeze et al., 1995; de Souza and Café-Filho, 2003; Zheng et al., 2013). High tolerances have been found in other Capsicum species like C. baccatum L., $C$. chinense Jacq., C. frutescens L., and C. pubescens Ruiz \& Pav. (Lee et al., 2001; de Souza and Café-Filho, 2003; Blat et al., 2005 and 2006). Despite $C$. chinense and $C$. frutescens are grouped within the $C$. annuum botanical complex, sexual incompatibility (both pre and postzygotic) has been reported on many occasions, depending on the accession (Manzur et al., 2015). Therefore, the introgression of genes from other species into C. annuum is limited. Recently, McCoy and Bosland (2019) evaluated 152 accessions of Capsicum spp. under natural (not controlled) infection conditions in New Mexico fields. They putatively identified several $C$. annuum accessions and one accession of chiltepin (C. annuum var. glabriusculum (Dunal)), opening the possibility to find resistance genes in $C$. annuum. In this regard, screenings for PM resistance have not been conducted yet with sufficient accessions of $C$. annuum var. glabriusculum, a wild and semi-wild form of pepper, which has been described as a close ancestor of $C$. annuum (Aguilar-Meléndez et al., 2009; Kraft et al., 2013; HayanoKanashiro et al., 2016; Pereira et al., 2019). Several populations of wild and semiwild peppers are naturally distributed across Mexico (Luna-Ruiz et al., 2018), and Kraft et al. (2013) assembled a collection of wild and semiwild peppers from Mexico and Southern USA, which may be good candidates to explore for additional sources of resistance to PM.

The objective of the present work was to screen, under controlled conditions, a wide range of Capsicum spp. accessions, including $C$. annuum var. glabriusculum, to identify possible novel sources of tolerance or resistance to PM. 


\section{Materials and Methods}

\section{Plant material and infection assay}

Forty-nine accessions of five different Capsicum taxa $(22$ C. annuum including some commercial cultivars, 5 C. annuum var. glabriusculum, 13 C. baccatum, 6 C. chinense, 1 C. frutescens. and 2 C. pubescens) were evaluated in two different trials (Table 1). In each trial three commercial accessions known for their good response against $\mathrm{PM}$ in field conditions (C. annuum: $\left.\mathrm{Co}-0^{*}\right)$ were included as controls. In addition, in the second trial four accessions selected from the first trial were re-evaluated to confirm their response against PM.

Table 1. Plant materials used, accessions names, abbreviations, origins and number of replicates in each trial tested

\begin{tabular}{|c|c|c|c|c|}
\hline Accession & Abbreviation & Origin & \multicolumn{2}{|c|}{ No. replicates by trial } \\
\hline \multicolumn{3}{|c|}{ Capsicum annuum } & First & Second \\
\hline Ací Sivri & A-09 & Turkey & - & 5 \\
\hline Acorde RZ F $F_{1}(53-142)$ & Co-01 & Rijk Zwaan, Spain & 20 & 5 \\
\hline Ancho 101 & A-10 & Mexico, USA & - & 5 \\
\hline Ancho Mulato & A-17 & Mexico, USA & - & 5 \\
\hline Bola & A-01 & Murcia, Spain & 5 & - \\
\hline California Wonder (r) & A-23 & $\begin{array}{c}\text { Breeding line COMAV, } \\
\text { Valencia, Spain }\end{array}$ & - & 5 \\
\hline California Wonder (y) & A-02 & $\begin{array}{c}\text { Breeding line, COMAV, } \\
\text { Valencia, Spain }\end{array}$ & 5 & - \\
\hline Carmagnola Rosso & A-12 & Carmagnola, Italy & - & 5 \\
\hline Chile Serrano 204D & A-03 & Mexico/USA & 5 & - \\
\hline Chile Serrano & A-04 & Mexico/USA & 5 & - \\
\hline Jalapeño Candelaria & A-13 & Mexico/USA & - & 5 \\
\hline Jalapeño Espinalteco 10397 & A-21 & Mexico/USA & - & 5 \\
\hline Jalapeño M. & A-18 & Mexico/USA & - & 5 \\
\hline Kabuki F $_{1}(\mathrm{BF} 50820)$ & Co-02 & Syngenta, Spain & 20 & 5 \\
\hline Largo de Reus & A-05 & Mascarell Seed Co., Spain & 5 & - \\
\hline Largo Valenciano & A-14 & Alicante, Spain & - & 5 \\
\hline Mojo Palmero & A-22 & La Palma, Spain & - & 5 \\
\hline Nirvin RZ F $F_{1}(35-150)$ & Co-03 & Rijk Zwaan, Spain & 10 & 5 \\
\hline Pasilla Bajío & A-06 & Mexico/USA & 5 & - \\
\hline Pimiento Valenciano & A-16 & Valencia, Spain & - & 5 \\
\hline Piquillo & A-07 & Navarra, Spain & 5 & - \\
\hline Serrano Criollo Morelos & A-08 & Morelos, Mexico & 5 & - \\
\hline \multicolumn{5}{|c|}{ C. annuum var. glabriusculum } \\
\hline Arizona 1003 & $\mathrm{Ag}-01$ & Arizona, USA & 5 & 5 \\
\hline Chiapas 1333 & $\mathrm{Ag}-03$ & Chiapas, Mexico & - & 5 \\
\hline Nayarit 1411 & $\mathrm{Ag}-04$ & Nayarit, Mexico & - & 5 \\
\hline Oaxaca 1430 & Ag-02 & Oaxaca, Mexico & 5 & 5 \\
\hline Veracruz 1196 & Ag-06 & Veracruz, Mexico & - & 5 \\
\hline \multicolumn{5}{|c|}{ C. baccatum } \\
\hline Bol - 103 & B-04 & Santa Cruz, Bolivia & 5 & - \\
\hline Bol - 104 & B-05 & Santa Cruz, Bolivia & 5 & - \\
\hline Bol - 106 & B-11 & Santa Cruz, Bolivia & - & 5 \\
\hline Bol - 108 & B-12 & Santa Cruz, Bolivia & - & 5 \\
\hline Bol - 111 & B-13 & Santa Cruz, Bolivia & - & 5 \\
\hline Bol - 115 & B-14 & Santa Cruz, Bolivia & - & 5 \\
\hline Bol - 117 & B-07 & Santa Cruz, Bolivia & 5 & - \\
\hline
\end{tabular}




\begin{tabular}{|c|c|c|c|c|}
\hline $\mathrm{Bol}-120$ & B-15 & Santa Cruz, Bolivia & - & 5 \\
\hline Bol - 134 & B-01 & Santa Cruz, Bolivia & 5 & - \\
\hline $\mathrm{Bol}-37 \mathrm{R}$ & B-03 & Chuquisaca, Bolivia & 5 & - \\
\hline Asta de Toro & B-02 & Cochabamba, Bolivia & 5 & - \\
\hline Bol - 67 & B-09 & Cochabamba, Bolivia & 5 & - \\
\hline Bol -71 & B-10 & Santa Cruz, Bolivia & 5 & 5 \\
\hline \multicolumn{5}{|c|}{ C. chinense } \\
\hline Aji dulce & C-01 & Venezuela & 5 & - \\
\hline Bol - 198 & C-02 & Santa Cruz, Bolivia & 5 & 5 \\
\hline Ají Charapita & C-03 & Amazonia, Peru & 5 & - \\
\hline ECU-994 & C-04 & Ecuador & 5 & - \\
\hline Habanero Rojo & C-06 & Pennsylvania, USA & - & 5 \\
\hline Ají Mochero & $\mathrm{C}-05$ & Valle de Moche, Peru & 5 & - \\
\hline \multicolumn{5}{|c|}{ C. frutescens } \\
\hline Bol - 144 & F-01 & Santa Cruz, Bolivia & 5 & - \\
\hline \multicolumn{5}{|c|}{ C.pubescens } \\
\hline Bol - 188 & P-01 & Chuquisaca, Bolivia & - & 5 \\
\hline Bol - 59 & $\mathrm{P}-02$ & Cochabamba, Bolivia & - & 5 \\
\hline
\end{tabular}

Seeds were sown in trays and one month later seedlings were transplanted to $7.5 \times 7.5 \mathrm{~cm}$ pots. One week after transplanting, plants were trimmed to leave only three to four basal leaves (Figure 1A). Plants were then placed in mini greenhouses in a randomized block design (Figure 1B). Mini greenhouses were placed in climate chambers with an average temperature of $21^{\circ} \mathrm{C} \pm 2$, and relative humidity of $80 \% \pm 5$ (Bai et al., 2003; Zheng et al., 2012).

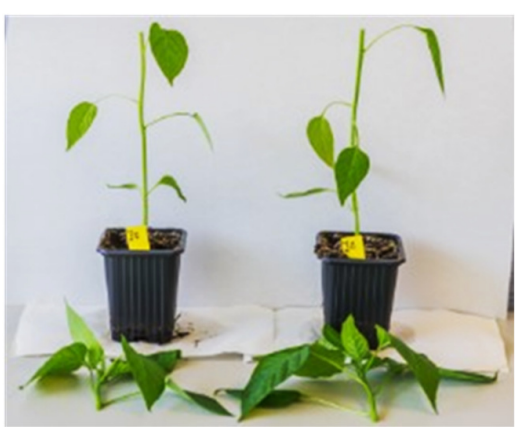

(A)

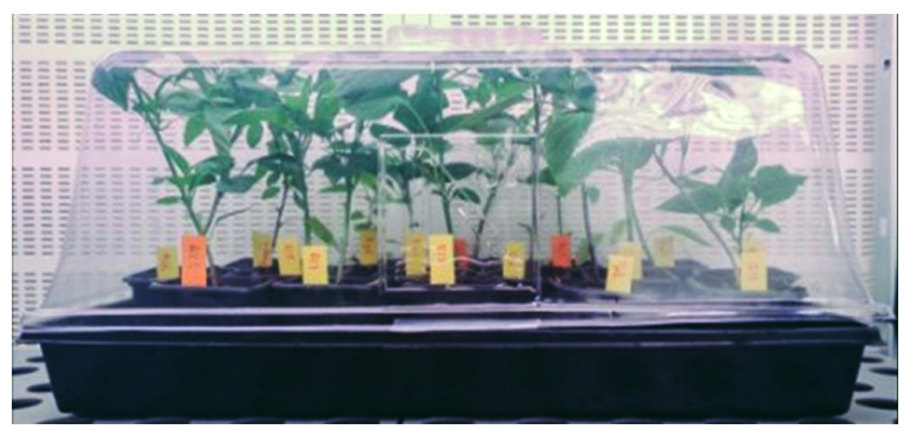

(B)

Figure 1. Experiment setup

(A) 30 days old pepper plants decapitated ready to be inoculated; (B) mini greenhouses used to avoid spreading over inside climatic chamber of inoculum

Plants were inoculated and later reinoculated three times (at 7,14 and 21 days after the first inoculation) by spraying the abaxial part of the leaves with a water suspension of $10^{4}$ conidia $\mathrm{ml}^{-1}$ (maximum concentration obtained). L. taurica inoculum was obtained from infected leaves of peppers grown in commercial fields and kept in vivo in susceptible materials under controlled conditions. Plants were evaluated 30 and 60 days after the first inoculation (DAI).

\section{Response evaluation}

Due to the broad range of Capsicum species evaluated and to overcome the possible bias, a Composite Infection Index (CII) based in Daubeze et al. (1995) was used. The total number of leaves (TL) and the total number of leaves affected (LA) showing chlorotic spots were recorded in each evaluation date. Also, a LA/TL ratio, similar to "the sporulation intensity" of Daubeze, was calculated. The area affected in the most damaged 
leaf, named maximum area affected (MAA), was also considered as Daubeze's "leaf area per plant". The MAA was recorded visually by scoring the proportion of leaf surface covered by chlorotic or sporulation spots (from 0.00 to 1.00 was used where $10 \%$ surface of chlorotic-sporulate spots was $0.10,20 \%=0.20 \ldots$ and $100 \%=1.00$ ). Our proposed CII (on a 0.00-1.00 scale) was calculated as follows:

$$
\mathrm{CII}=\frac{\left(\frac{\mathrm{LA}}{\mathrm{TL}}\right)+\mathrm{MAA}}{2}
$$

\section{Statistical analysis}

Five plants per accession were evaluated in both trials, except commercial accessions in the first trial (10 to 20 plants). Two-way factorial analysis of variance (ANOVA, $\mathrm{P}<0.05$ ) was performed using individual plant $\mathrm{n}$-values to assess accession and block (mini greenhouses) effects (Hoshmand, 2020). In addition, StudentNewman-Keuls post-hoc multiple range test $(\mathrm{P}<0.05)$ was used to detect significant differences among accessions means for all the evaluated traits. Data was standardized by z-score to compare within and between trials. All statistical analysis was performed using Statgraphics Centurion XVIII (18.1.13 ver., Statgraphics Technologies, VA, USA).

\section{Results}

\section{First screening}

The first trial infection produced the characteristic damage of PM in the plants. When assessing the performance of the 27 accessions tested in this trial, the ANOVA showed significant differences among them for all the evaluated traits $(\mathrm{P}<0.001$, Suppl. Table 1$)$. As plants were organized in a randomized block design, the possible influence of the block was investigated. Also, the effect of the block contributed significantly to variation (Suppl. Table 1). This circumstance was considered on post-hoc analysis.

Infection intensity was higher at 30 than at $60 \mathrm{DAI}$ (Table 2). Average LA TL ${ }^{-1}$ ratio was 0.32 at 30 DAI and decreased to 0.18 at $60 \mathrm{DAI}$; average MAA was 0.18 at $30 \mathrm{DAI}$ and 0.23 at $60 \mathrm{DAI}$; average CII was 0.25 at $30 \mathrm{DAI}$ and 0.20 at $60 \mathrm{DAI}$.

At 30 DAI the mean values of total number of leaves (TL) ranged from 7.85 (Co-02) to 20.38 (C-03), and accessions separate significantly in different groups, being Co-02, Co-01, A-02, A-07, Co-03, A-05 and B10 the ones with the lowest number of leaves, whereas A-08, B-04, F-01, Ag-02, and C-03 showed the highest number (Table 2). The average number of leaves affected (LA) ranged from 0.36 (Ag-01) to 8.40 (C-03) and the accession Ag-01 differed significantly from the six most affected accessions (i.e., B-07, B-03, B-01, A-04, A08 and C-03). For the ratio of affected leaves, mean values ranged from 0.07 (Ag-01) to 0.56 (A-02) and Ag-01 and C-05 significantly differed from the last three accessions (A-08, B-01 and A-02). For the MAA, mean values were comprised from 0.04 (Ag-01) to 0.42 (A-04), although only Ag-01 and C-05 were differed significantly from the two worst two accessions, A-02 and A-04. In the case of the composite infection index (CII), mean values ranged from 0.05 (Ag-01) as the most tolerant, to 0.44 (A-04), as the most susceptible. In this regard, the statistical test separates clearly Ag-01 and C-05 from the worst five accessions (B-10, B-01, A08, A-02 and A-04) (Table 2).

At 60 DAI, the ANOVA detected significant differences among accessions in most traits, particularly in TL and LA (Suppl. Table 2). In TL, accessions grouped significantly like those at $30 \mathrm{DAI}$ and, thus, the accessions with the highest number of leaves at $30 \mathrm{DAI}$ also had high number of leaves at $60 \mathrm{DAI}$ (Table 2). By contrast, this was not the case for the rest of parameters, indicating that the evolution of these traits from 30 to $60 \mathrm{DAP}$ was different among the accessions. Thus, some accessions like A-06, Ag-01 and Ag-02 evolved with very low values of LA, LA/TL ratio, MAA and CII, C- 02 showed moderate values in both 30 and $60 \mathrm{DAP}$, and C-03 and B-10 continued with high disease values (Table 2). On the contrary, some accessions like A-08, B-01, 
B-03, A-04, B-05, which had very bad values in LA, LA/TL ratio, MAA and CII at $30 \mathrm{DAI}$, turned into very good performance at $60 \mathrm{DAI}$ (Table 2).

Table 2. First trial mean values of total number of leaves (TL), leaves affected (LA), LA/TL ratio, maximum area affected (MAA) and composite infection index (CII) at 30 and 60 DAI

\begin{tabular}{|c|c|c|c|c|c|c|c|c|c|c|}
\hline \multirow{2}{*}{ Acces. } & \multicolumn{2}{|c|}{ TL } & \multicolumn{2}{|c|}{ LA } & \multicolumn{2}{|c|}{ LA/TL ratio } & \multicolumn{2}{|c|}{ MAA } & \multicolumn{2}{|c|}{ CII } \\
\hline & $30 \mathrm{DAI}$ & $60 \mathrm{DAI}$ & $30 \mathrm{DAI}$ & $60 \mathrm{DAI}$ & $30 \mathrm{DAI}$ & $60 \mathrm{DAI}$ & $30 \mathrm{DAI}$ & $60 \mathrm{DAI}$ & $30 \mathrm{DAI}$ & $60 \mathrm{DAI}$ \\
\hline \multicolumn{11}{|c|}{ Capsicum annuum } \\
\hline A-01 & $12.02 \mathrm{ABCD}$ & $21.03 \mathrm{ABCDE}$ & $3.40 \mathrm{ABC}$ & $3.47 \mathrm{AB}$ & $0.30_{\mathrm{ABC}}$ & $0.17_{\mathrm{A}}$ & $0.13_{\mathrm{AB}}$ & $0.21_{\mathrm{A}}$ & $0.22 \mathrm{ABCD}$ & $0.19_{\mathrm{A}}$ \\
\hline $\mathrm{A}-02$ & $9.12_{\mathrm{AB}}$ & $14.91_{\mathrm{AB}}$ & $4.28 \mathrm{ABC}$ & $3.29 \mathrm{AB}$ & $0.56 \mathrm{C}$ & $0.22_{\mathrm{A}}$ & $0.30_{\mathrm{BC}}$ & $0.21_{\mathrm{A}}$ & $0.43 \mathrm{D}$ & $0.21_{\mathrm{A}}$ \\
\hline A-03 & $12.64 \mathrm{ABCDE}$ & 23.30 ABCDEF & $3.54 \mathrm{ABC}$ & $4.30_{\mathrm{AB}}$ & $0.29 \mathrm{ABC}$ & $0.17_{\mathrm{A}}$ & $0.18_{\mathrm{AB}}$ & $0.41_{\mathrm{A}}$ & $0.23 \mathrm{ABCD}$ & $0.29 \mathrm{~A}$ \\
\hline $\mathrm{A}-04$ & $16.56 \mathrm{CDEFG}$ & $40.10_{\mathrm{G}}$ & $6.46 \mathrm{CD}$ & $3.70_{\mathrm{AB}}$ & $0.45 \mathrm{ABC}$ & $0.11_{\mathrm{A}}$ & $0.42 \mathrm{C}$ & $0.17 \mathrm{~A}$ & $0.44 \mathrm{D}$ & $0.14 \mathrm{~A}$ \\
\hline $\mathrm{A}-05$ & $9.52_{\mathrm{AB}}$ & $16.23 \mathrm{ABC}$ & $3.19 \mathrm{ABC}$ & $3.22 \mathrm{AB}$ & $0.33 \mathrm{ABC}$ & $0.19_{\mathrm{A}}$ & $0.11_{\mathrm{AB}}$ & $0.36 \mathrm{~A}$ & $0.22 \mathrm{ABCD}$ & $0.27 \mathrm{~A}$ \\
\hline A-06 & 13.68 ABCDEF & 21.77 ABCDEF & $2.41 \mathrm{ABC}$ & $2.58_{\mathrm{A}}$ & $0.19_{\mathrm{ABC}}$ & $0.11_{\mathrm{A}}$ & $0.13_{\mathrm{AB}}$ & $0.12_{\mathrm{A}}$ & $0.16_{\mathrm{ABCD}}$ & $0.12_{\mathrm{A}}$ \\
\hline $\mathrm{A}-07$ & $9.17 \mathrm{AB}$ & $13.71_{\mathrm{A}}$ & $2.84 \mathrm{ABC}$ & $2.71_{\mathrm{A}}$ & $0.30 \mathrm{ABC}$ & $0.18_{\mathrm{A}}$ & $0.11_{\mathrm{AB}}$ & $0.34 \mathrm{~A}$ & $0.20 \mathrm{ABCD}$ & $0.26 \mathrm{~A}$ \\
\hline A-08 & 17.43 DEFG & $35.49 \mathrm{EFG}$ & $8.16 \mathrm{D}$ & $2.29 \mathrm{~A}$ & $0.51 \mathrm{BC}$ & $0.10_{\mathrm{A}}$ & $0.25 \mathrm{ABC}$ & $0.10_{\mathrm{A}}$ & $0.38 \mathrm{D}$ & $0.10_{\mathrm{A}}$ \\
\hline Co-01 & $9.00_{\mathrm{AB}}$ & $15.85 \mathrm{ABC}$ & $2.95 \mathrm{ABC}$ & $3.25 \mathrm{AB}$ & $0.35 \mathrm{ABC}$ & $0.20_{\mathrm{A}}$ & $0.15_{\mathrm{AB}}$ & $0.24 \mathrm{~A}$ & $0.25 \mathrm{ABCD}$ & $0.22_{\mathrm{A}}$ \\
\hline Co-02 & $7.85 \mathrm{~A}$ & $13.95 \mathrm{~A}$ & $3.40 \mathrm{ABC}$ & $3.25 \mathrm{AB}$ & $0.45 \mathrm{ABC}$ & $0.25 \mathrm{~A}$ & $0.14 \mathrm{AB}$ & $0.18_{\mathrm{A}}$ & $0.30_{\mathrm{ABCD}}$ & $0.21_{\mathrm{A}}$ \\
\hline Co-03 & $9.20_{\mathrm{AB}}$ & $14.70_{\mathrm{AB}}$ & $3.10 \mathrm{ABC}$ & $3.60 \mathrm{AB}$ & $0.35 \mathrm{ABC}$ & $0.27 \mathrm{~A}$ & $0.27 \mathrm{ABC}$ & $0.21_{\mathrm{A}}$ & $0.31 \mathrm{ABCD}$ & $0.24 \mathrm{~A}$ \\
\hline \multicolumn{11}{|c|}{ C. annuum var. glabriusculum } \\
\hline Ag-01 & 14.92 ВCDEFG & $30.01 \mathrm{CDEFG}$ & $0.36 \mathrm{~A}$ & $2.50_{\mathrm{A}}$ & $0.07 \mathrm{~A}$ & $0.05 \mathrm{~A}$ & $0.04_{\mathrm{A}}$ & $0.13 \mathrm{~A}$ & $0.05 \mathrm{~A}$ & $0.09 \mathrm{~A}$ \\
\hline Ag-02 & $19.08 \mathrm{FG}$ & $36.59 \mathrm{FG}$ & $2.64 \mathrm{ABC}$ & $5.50_{\mathrm{AB}}$ & $0.10_{\mathrm{AB}}$ & $0.19_{\mathrm{A}}$ & $0.08_{\mathrm{AB}}$ & $0.17_{\mathrm{A}}$ & $0.09 \mathrm{ABC}$ & $0.18_{\mathrm{A}}$ \\
\hline \multicolumn{11}{|c|}{ C. baccatum } \\
\hline B-01 & $12.56 \mathrm{ABCDE}$ & 22.84 ABCDEF & $5.73 \mathrm{BCD}$ & $4.24 \mathrm{AB}$ & $0.53 \mathrm{C}$ & $0.18_{\mathrm{A}}$ & $0.22 \mathrm{ABC}$ & $0.11_{\mathrm{A}}$ & $0.37 \mathrm{D}$ & $0.14 \mathrm{~A}$ \\
\hline B-02 & $16.64 \mathrm{CDEFG}$ & $36.56 \mathrm{FG}$ & $2.67 \mathrm{ABC}$ & $4.96_{\mathrm{AB}}$ & $0.17 \mathrm{ABC}$ & $0.20_{\mathrm{A}}$ & $0.16_{\mathrm{AB}}$ & $0.29 \mathrm{~A}$ & $0.17 \mathrm{ABCD}$ & $0.24_{\mathrm{A}}$ \\
\hline B-03 & 13.92 ABCDEF & 24.09 ABCDEF & $5.66 \mathrm{BCD}$ & $2.91_{\mathrm{A}}$ & $0.38_{\mathrm{ABC}}$ & $0.12_{\mathrm{A}}$ & $0.28 \mathrm{ABC}$ & $0.16_{\mathrm{A}}$ & $0.33 \mathrm{BCD}$ & $0.14 \mathrm{~A}$ \\
\hline B-04 & 18.28 DEFG & 30.91 DEFG & $3.54 \mathrm{ABC}$ & $4.49 \mathrm{AB}$ & $0.21 \mathrm{ABC}$ & $0.29 \mathrm{~A}$ & $0.16_{\mathrm{AB}}$ & $0.26 \mathrm{~A}$ & $0.18 \mathrm{ABCD}$ & $0.27 \mathrm{~A}$ \\
\hline B-05 & $10.84_{\mathrm{ABC}}$ & 25.65 ABCDEFG & $3.16_{\mathrm{ABC}}$ & $3.27_{\mathrm{AB}}$ & $0.37 \mathrm{ABC}$ & $0.14 \mathrm{~A}$ & $0.26 \mathrm{ABC}$ & $0.17_{\mathrm{A}}$ & $0.32 \mathrm{ABCD}$ & $0.16_{\mathrm{A}}$ \\
\hline B-07 & $16.83 \mathrm{CDEFG}$ & 32.06 DEFG & $5.27 \mathrm{BCD}$ & $5.93 \mathrm{AB}$ & $0.34 \mathrm{ABC}$ & $0.19 \mathrm{~A}$ & $0.16_{\mathrm{AB}}$ & $0.39 \mathrm{~A}$ & $0.25 \mathrm{ABCD}$ & $0.29 \mathrm{~A}$ \\
\hline B-09 & 14.48 вCDEFG & 34.74 DEFG & $3.76 \mathrm{ABC}$ & $4.58 \mathrm{AB}$ & $0.29 \mathrm{ABC}$ & $0.12_{\mathrm{A}}$ & $0.21 \mathrm{ABC}$ & $0.25 \mathrm{~A}$ & $0.25 \mathrm{ABCD}$ & $0.19_{\mathrm{A}}$ \\
\hline B-10 & $10.12 \mathrm{AB}$ & $19.86 \mathrm{ABCD}$ & $4.44 \mathrm{ABC}$ & $4.62 \mathrm{AB}$ & $0.48 \mathrm{ABC}$ & $0.25 \mathrm{~A}$ & $0.21 \mathrm{AB}$ & $0.37 \mathrm{~A}$ & $0.34 \mathrm{CD}$ & $0.31_{\mathrm{A}}$ \\
\hline \multicolumn{11}{|c|}{ C. chinense } \\
\hline C-01 & 12.85 ABCDEF & 25.08 ABCDEF & $2.64 \mathrm{ABC}$ & $3.58 \mathrm{AB}$ & $0.20 \mathrm{ABC}$ & $0.15 \mathrm{~A}$ & $0.14_{\mathrm{AB}}$ & $0.22 \mathrm{~A}$ & $0.17 \mathrm{ABCD}$ & $0.18_{\mathrm{A}}$ \\
\hline C-02 & 13.35 ABCDEF & $12.72 \mathrm{~A}$ & $3.36 \mathrm{ABC}$ & $4.02 \mathrm{AB}$ & $0.26 \mathrm{ABC}$ & $0.29 \mathrm{~A}$ & $0.12 \mathrm{AB}$ & $0.22 \mathrm{~A}$ & $0.19 \mathrm{ABCD}$ & $0.25 \mathrm{~A}$ \\
\hline C-03 & $20.38 \mathrm{G}$ & $35.39 \mathrm{EFG}$ & $8.40 \mathrm{D}$ & 7.43 в & $0.41 \mathrm{ABC}$ & $0.20 \mathrm{~A}$ & $0.20_{\mathrm{AB}}$ & $0.27 \mathrm{~A}$ & $0.30 \mathrm{ABCD}$ & $0.24 \mathrm{~A}$ \\
\hline C-04 & $16.48 \mathrm{CDEFG}$ & 28.88 BCDEFG & $4.12 \mathrm{ABC}$ & $5.22 \mathrm{AB}$ & $0.31_{\mathrm{ABC}}$ & $0.17 \mathrm{~A}$ & $0.14_{\mathrm{AB}}$ & $0.17 \mathrm{~A}$ & $0.23 \mathrm{ABCD}$ & $0.17 \mathrm{~A}$ \\
\hline C-05 & $17.14 \mathrm{CDEFG}$ & $29.80 \mathrm{CDEFG}$ & $1.86 \mathrm{AB}$ & $4.86 \mathrm{AB}$ & $0.08_{\mathrm{A}}$ & $0.20_{\mathrm{A}}$ & $0.04_{\mathrm{A}}$ & $0.19 \mathrm{~A}$ & $0.06_{\mathrm{AB}}$ & $0.19_{\mathrm{A}}$ \\
\hline \multicolumn{11}{|c|}{ C. frutescens } \\
\hline F-01 & $18.79 \mathrm{EFG}$ & 31.28 DEFG & $3.91 \mathrm{ABC}$ & $5.74_{\mathrm{AB}}$ & $0.24_{\mathrm{ABC}}$ & $0.17_{\mathrm{A}}$ & $0.14_{\mathrm{AB}}$ & $0.27 \mathrm{~A}$ & $0.19 \mathrm{ABCD}$ & $0.22_{\mathrm{A}}$ \\
\hline Mean & 13.81 & 25.46 & 3.90 & 4.06 & 0.32 & 0.18 & 0.18 & 0.23 & 0.25 & 0.20 \\
\hline
\end{tabular}

\section{Second screening}

Based on the results of the first screening, four accessions were selected to be included in the second screening, as they showed good response (Ag- 01 and $\mathrm{Ag}-02$ ), moderate response (C-02) and bad response (B10), in both 30 and $60 \mathrm{DAI}$ evaluations. Unfortunately, accession A- 06 which showed a good performance in the first screening and was planned to be included as well, showed a poor germination $(<10 \%)$ and, consequently, it was discarded for the second screening.

The ANOVA found significant differences between accessions in most traits, with the only exception of MAA, and in most cases the effect of the block was also significant, which was considered when doing posthoc analysis (Suppl. Table 3). On the whole, the severity of the symptoms at $30 \mathrm{DAI}$ in all the accessions was higher on this second screening than those recorded in the first trial (Tables 2 and 3; Figure 2). 
Table 3. Second trial mean values of total number of leaves (TL), leaves affected (LA), LA/TL ratio, maximum area affected (MAA) and composite infection index (CII) at 30 and 60 DAI

\begin{tabular}{|c|c|c|c|c|c|c|c|c|c|c|}
\hline \multirow{2}{*}{ Acces. } & \multicolumn{2}{|c|}{ TL } & \multicolumn{2}{|c|}{ LA } & \multicolumn{2}{|c|}{ LA/TL ratio } & \multicolumn{2}{|c|}{ MAA } & \multicolumn{2}{|c|}{ CII } \\
\hline & $30 \mathrm{DAI}$ & $60 \mathrm{DAI}$ & $30 \mathrm{DAI}$ & $60 \mathrm{DAI}$ & $30 \mathrm{DAI}$ & $60 \mathrm{DAI}$ & $30 \mathrm{DAI}$ & $60 \mathrm{DAI}$ & $30 \mathrm{DAI}$ & $60 \mathrm{DAI}$ \\
\hline \multicolumn{11}{|c|}{ Capsicum annuum } \\
\hline A-09 & $7.04 \mathrm{~A}$ & $10.59 \mathrm{~A}$ & $6.03 \mathrm{ABC}$ & $4.77_{\mathrm{AB}}$ & $0.85 \mathrm{ABC}$ & $0.62_{\mathrm{D}}$ & $0.42_{\mathrm{A}}$ & $0.18_{\mathrm{A}}$ & $0.64_{\mathrm{AB}}$ & $0.40_{\mathrm{B}}$ \\
\hline A-10 & $10.18 \mathrm{AB}$ & $21.13 \mathrm{AB}$ & $5.70 \mathrm{ABC}$ & $7.63 \mathrm{AB}$ & $0.59 \mathrm{ABC}$ & $0.36 \mathrm{ABC}$ & $0.34 \mathrm{~A}$ & $0.20_{\mathrm{A}}$ & $0.47_{\mathrm{AB}}$ & $0.28 \mathrm{AB}$ \\
\hline A-12 & $5.84 \mathrm{~A}$ & $19.87 \mathrm{AB}$ & $3.75 \mathrm{ABC}$ & $4.24 \mathrm{AB}$ & $0.73 \mathrm{ABC}$ & $0.21_{\mathrm{ABC}}$ & $0.25 \mathrm{~A}$ & $0.14 \mathrm{~A}$ & $0.49 \mathrm{AB}$ & $0.18_{\mathrm{AB}}$ \\
\hline A-13 & $9.60 \mathrm{AB}$ & $22.04 \mathrm{AB}$ & $7.51_{\mathrm{ABC}}$ & $5.96 \mathrm{AB}$ & $0.74 \mathrm{ABC}$ & $0.27 \mathrm{ABC}$ & $0.37 \mathrm{~A}$ & $0.22_{\mathrm{A}}$ & $0.56_{\mathrm{AB}}$ & $0.24 \mathrm{AB}$ \\
\hline A-14 & $5.93 \mathrm{~A}$ & $18.31 \mathrm{AB}$ & $4.58 \mathrm{ABC}$ & $6.49 \mathrm{AB}$ & $0.84 \mathrm{ABC}$ & $0.44 \mathrm{BCD}$ & $0.30 \mathrm{~A}$ & $0.22_{\mathrm{A}}$ & $0.57 \mathrm{AB}$ & $0.33 \mathrm{AB}$ \\
\hline A-16 & $3.57 \mathrm{~A}$ & $18.43 \mathrm{AB}$ & $2.48_{\mathrm{AB}}$ & $3.94 \mathrm{AB}$ & $0.93 \mathrm{ABC}$ & $0.24 \mathrm{ABC}$ & $0.48_{\mathrm{A}}$ & $0.13_{\mathrm{A}}$ & $0.70_{\mathrm{AB}}$ & $0.18_{\mathrm{AB}}$ \\
\hline A-17 & $8.24 \mathrm{~A}$ & $25.27 \mathrm{AB}$ & $5.95 \mathrm{ABC}$ & $9.04 \mathrm{AB}$ & $0.78 \mathrm{ABC}$ & $0.35 \mathrm{ABC}$ & $0.35 \mathrm{~A}$ & $0.22 \mathrm{~A}$ & $0.56_{\mathrm{AB}}$ & $0.29 \mathrm{AB}$ \\
\hline A-18 & $7.70_{\mathrm{A}}$ & $19.77 \mathrm{AB}$ & $5.10_{\mathrm{ABC}}$ & $5.10_{\mathrm{AB}}$ & $0.77_{\mathrm{ABC}}$ & $0.40_{\mathrm{ABCD}}$ & $0.39 \mathrm{~A}$ & $0.41_{\mathrm{B}}$ & $0.58_{\mathrm{AB}}$ & $0.41_{\mathrm{B}}$ \\
\hline A-21 & $8.55 \mathrm{~A}$ & $17.69 \mathrm{AB}$ & $7.38 \mathrm{ABC}$ & $6.65_{\mathrm{AB}}$ & $0.95 \mathrm{BC}$ & $0.46 \mathrm{CD}$ & $0.55 \mathrm{~A}$ & $0.17_{\mathrm{A}}$ & 0.75 в & $0.31_{\mathrm{AB}}$ \\
\hline A-22 & $10.35_{\mathrm{AB}}$ & $26.56_{\mathrm{ABC}}$ & $8.59 \mathrm{ABCD}$ & $5.97 \mathrm{AB}$ & $0.86_{\mathrm{ABC}}$ & $0.23 \mathrm{ABC}$ & $0.44 \mathrm{~A}$ & $0.24_{\mathrm{A}}$ & $0.65_{\mathrm{AB}}$ & $0.23_{\mathrm{AB}}$ \\
\hline A-23 & $5.33 \mathrm{~A}$ & $18.86 \mathrm{AB}$ & $2.90 \mathrm{ABC}$ & $3.07 \mathrm{AB}$ & $0.52 \mathrm{AB}$ & $0.17 \mathrm{ABC}$ & $0.18_{\mathrm{A}}$ & $0.17 \mathrm{~A}$ & $0.35 \mathrm{~A}$ & $0.17_{\mathrm{AB}}$ \\
\hline $\mathrm{Co}-01$ & $7.15 \mathrm{~A}$ & $21.91 \mathrm{AB}$ & $5.36 \mathrm{ABC}$ & $4.46 \mathrm{AB}$ & $0.74 \mathrm{ABC}$ & $0.20_{\mathrm{ABC}}$ & $0.43 \mathrm{~A}$ & $0.09_{\mathrm{A}}$ & $0.58_{\mathrm{AB}}$ & $0.14_{\mathrm{A}}$ \\
\hline $\mathrm{Co}-02$ & $3.59 \mathrm{~A}$ & $16.37 \mathrm{AB}$ & $2.29 \mathrm{~A}$ & $4.27_{\mathrm{AB}}$ & $0.75 \mathrm{ABC}$ & $0.24 \mathrm{ABC}$ & $0.25 \mathrm{~A}$ & $0.13_{\mathrm{A}}$ & $0.50_{\mathrm{AB}}$ & $0.19_{\mathrm{AB}}$ \\
\hline $\mathrm{Co}-03$ & $5.39 \mathrm{~A}$ & $20.67 \mathrm{AB}$ & $3.32 \mathrm{ABC}$ & $5.26_{\mathrm{AB}}$ & $0.82_{\mathrm{ABC}}$ & $0.27 \mathrm{ABC}$ & $0.33_{\mathrm{A}}$ & $0.20_{\mathrm{A}}$ & $0.57_{\mathrm{AB}}$ & $0.23_{\mathrm{AB}}$ \\
\hline \multicolumn{11}{|c|}{ C. annuum var. glabriusculum } \\
\hline $\mathrm{Ag}-01$ & $23.29 \mathrm{D}$ & $44.67 \mathrm{CD}$ & $10.40 \mathrm{CDE}$ & $8.09 \mathrm{AB}$ & $0.51_{\mathrm{A}}$ & $0.18_{\mathrm{ABC}}$ & $0.30_{\mathrm{A}}$ & $0.10_{\mathrm{A}}$ & $0.41_{\mathrm{AB}}$ & $0.14_{\mathrm{A}}$ \\
\hline $\mathrm{Ag}-02$ & $10.44 \mathrm{AB}$ & $29.92 \mathrm{ABCD}$ & $6.54 \mathrm{ABC}$ & $8.32 \mathrm{AB}$ & $0.71 \mathrm{ABC}$ & $0.29 \mathrm{ABC}$ & $0.25 \mathrm{~A}$ & $0.15 \mathrm{~A}$ & $0.48_{\mathrm{AB}}$ & $0.22 \mathrm{AB}$ \\
\hline $\mathrm{Ag}-03$ & $7.45 \mathrm{~A}$ & $29.08 \mathrm{ABCD}$ & $6.44 \mathrm{ABC}$ & $8.61_{\mathrm{AB}}$ & $0.89 \mathrm{ABC}$ & $0.28 \mathrm{ABC}$ & $0.42_{\mathrm{A}}$ & $0.12_{\mathrm{A}}$ & $0.65_{\mathrm{AB}}$ & $0.20_{\mathrm{AB}}$ \\
\hline $\mathrm{Ag}-04$ & $16.72 \mathrm{BC}$ & $30.34 \mathrm{ABCD}$ & $10.13 \mathrm{BCDE}$ & 9.73 в & $0.69 \mathrm{ABC}$ & $0.30 \mathrm{ABC}$ & $0.34 \mathrm{~A}$ & $0.18_{\mathrm{A}}$ & $0.51_{\mathrm{AB}}$ & $0.24_{\mathrm{AB}}$ \\
\hline $\mathrm{Ag}-06$ & $19.36 \mathrm{CD}$ & $73.11 \mathrm{E}$ & $15.37 \mathrm{E}$ & $14.18 \mathrm{C}$ & $0.80 \mathrm{ABC}$ & $0.22 \mathrm{ABC}$ & $0.33 \mathrm{~A}$ & $0.29_{\mathrm{AB}}$ & $0.57_{\mathrm{AB}}$ & $0.25 \mathrm{AB}$ \\
\hline \multicolumn{11}{|c|}{ C. baccatum } \\
\hline B-10 & $10.42 \mathrm{AB}$ & $33.17 \mathrm{BCD}$ & $7.45 \mathrm{ABC}$ & $6.19 \mathrm{AB}$ & $0.69 \mathrm{ABC}$ & $0.18 \mathrm{ABC}$ & $0.33 \mathrm{~A}$ & $0.21 \mathrm{~A}$ & $0.51_{\mathrm{AB}}$ & $0.19_{\mathrm{AB}}$ \\
\hline B-11 & $10.02_{\mathrm{AB}}$ & $29.07 \mathrm{ABCD}$ & $6.64 \mathrm{ABC}$ & $4.71 \mathrm{AB}$ & $0.75 \mathrm{ABC}$ & $0.18 \mathrm{ABC}$ & $0.26 \mathrm{~A}$ & $0.11_{\mathrm{A}}$ & $0.51_{\mathrm{AB}}$ & $0.15 \mathrm{~A}$ \\
\hline B-12 & $11.48_{\mathrm{AB}}$ & $26.15_{\mathrm{ABC}}$ & $9.29 \mathrm{ABCD}$ & $5.33_{\mathrm{AB}}$ & $0.77_{\mathrm{ABC}}$ & $0.19_{\mathrm{ABC}}$ & $0.50_{\mathrm{A}}$ & $0.29_{\mathrm{AB}}$ & $0.63_{\mathrm{AB}}$ & $0.24 \mathrm{AB}$ \\
\hline B-13 & $10.88 \mathrm{AB}$ & $33.44 \mathrm{BCD}$ & $10.38 \mathrm{CDE}$ & $3.33 \mathrm{AB}$ & $0.94 \mathrm{ABC}$ & $0.10_{\mathrm{AB}}$ & $0.37 \mathrm{~A}$ & $0.21 \mathrm{~A}$ & $0.65_{\mathrm{AB}}$ & $0.15 \mathrm{~A}$ \\
\hline B-14 & $21.42 \mathrm{CD}$ & $37.00_{\mathrm{BCD}}$ & $14.62 \mathrm{DE}$ & $6.96_{\mathrm{AB}}$ & $0.72 \mathrm{ABC}$ & $0.19_{\mathrm{ABC}}$ & $0.34 \mathrm{~A}$ & $0.16_{\mathrm{A}}$ & $0.53_{\mathrm{AB}}$ & $0.17_{\mathrm{AB}}$ \\
\hline B-15 & $11.65 \mathrm{AB}$ & $24.96 \mathrm{AB}$ & $7.17 \mathrm{ABC}$ & $6.04_{\mathrm{AB}}$ & $0.65 \mathrm{ABC}$ & $0.24 \mathrm{ABC}$ & $0.28_{\mathrm{A}}$ & $0.21_{\mathrm{A}}$ & $0.46_{\mathrm{AB}}$ & $0.22_{\mathrm{AB}}$ \\
\hline \multicolumn{11}{|c|}{ C. chinense } \\
\hline C-02 & $4.22 \mathrm{~A}$ & $17.87 \mathrm{AB}$ & $3.44 \mathrm{ABC}$ & $5.31_{\mathrm{AB}}$ & $0.97 \mathrm{C}$ & $0.34 \mathrm{ABC}$ & $0.36_{\mathrm{A}}$ & $0.27_{\mathrm{AB}}$ & $0.66_{\mathrm{AB}}$ & $0.31_{\mathrm{AB}}$ \\
\hline C-06 & $6.45 \mathrm{~A}$ & $26.81 \mathrm{ABC}$ & $5.45 \mathrm{ABC}$ & $5.85 \mathrm{AB}$ & $0.89 \mathrm{ABC}$ & 0.22 ABC & $0.41 \mathrm{~A}$ & $0.10_{\mathrm{A}}$ & $0.65_{\mathrm{AB}}$ & $0.16 \mathrm{AB}$ \\
\hline \multicolumn{11}{|c|}{ C.pubescens } \\
\hline P-01 & $10.98_{\mathrm{AB}}$ & $45.73 \mathrm{D}$ & $8.11_{\mathrm{ABC}}$ & $2.74 \mathrm{~A}$ & $0.85 \mathrm{ABC}$ & $0.10_{\mathrm{AB}}$ & $0.45 \mathrm{~A}$ & $0.15_{\mathrm{A}}$ & $0.65_{\mathrm{AB}}$ & $0.12_{\mathrm{A}}$ \\
\hline P-02 & $10.27 \mathrm{AB}$ & $37.02 \mathrm{BCD}$ & $6.19 \mathrm{ABC}$ & $3.20_{\mathrm{AB}}$ & $0.61 \mathrm{ABC}$ & $0.08_{\mathrm{A}}$ & $0.53 \mathrm{~A}$ & $0.16_{\mathrm{A}}$ & $0.57 \mathrm{AB}$ & $0.12_{\mathrm{A}}$ \\
\hline Mean & 9.78 & 27.44 & 6.85 & 6.05 & 0.77 & 0.26 & 0.36 & 0.19 & 0.57 & 0.22 \\
\hline
\end{tabular}




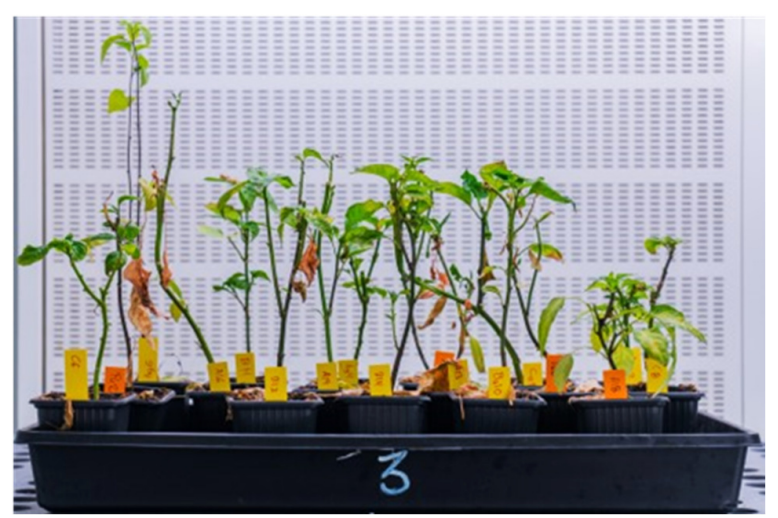

(A)

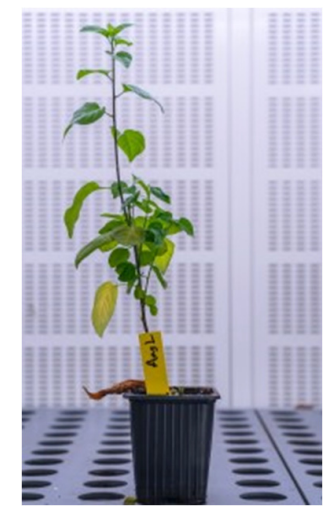

(B)

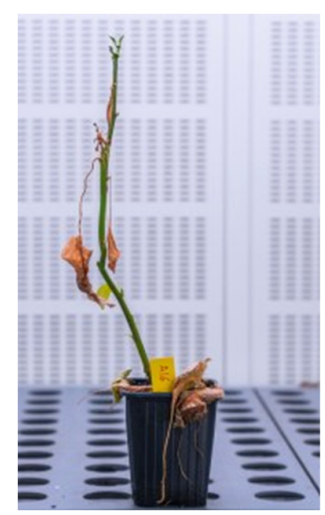

(C)

Figure 2. Results of the experiment; (A) Example tested plants; (B) Detail of Ag-01; (C) Detail of A-16 during the second trial at $30 \mathrm{DAI}$

TL mean values at $30 \mathrm{DAI}$ ranged from 3.57 (A-16) to 23.29 (Ag-01) and showed significant differences according to the ANOVA $(\mathrm{P}<0.000)$ (Suppl. Table 3). The statistical test separated a big group of fourteen accessions with the lowest values (A-16, Co-02, C-02, A-23, Co-03, A-12, A-14, C-06, A-09, Co-01, Ag-03, A18, A-17, and A-21) from another group of four $C$. annuum var. glabriusculum and C. baccatum accessions (Ag-01, Ag-04, Ag-06, and B-14) showing the highest TL values (Table 3). Regarding LA, significant differences were found among accessions (ANOVA, $\mathrm{P}<0.000$ ) (Suppl. Table 3 ) and mean values ranged from 2.29 (Co-02) to 15.37 (Ag-06). The accessions differed statistically in a similar way to TL, with Co-02 and A16 being the best accessions in one group and another group like the second group of TL (B-13, Ag-01, B-14 and Ag-06) (Table 3). The ANOVA also detected significant contribution of the accessions to the variation (P $<0.003$ ) in the LA/TL ratio (ANOVA) (Suppl. Table 3) and mean values were comprised between 0.51 (Ag01 ) and 0.97 (C-02) and the best accession Ag-01 differed significantly from the last two accessions (A-21 and C-02) (Table 3). As mentioned before, no significant differences were found in MAA (ANOVA, $P=0.350$ ) (Suppl. Table 3) and the statistical test did not show significant differences among accessions, although mean values ranged 0.18 (A-23) to 0.55 (A-21) (Table 3). Finally, significant differences among accessions were found in CII (ANOVA, $\mathrm{P}=0.018$ ) (Suppl. Table 3 ), with the mean values being comprised between 0.35 (the best accession A-23) and 0.75 (A-21), which differed significantly (Table 3).

In general, according to the values recorded at $60 \mathrm{DAI}$, plants looked recovered from inoculation (Table 3). The ANOVA found a significant effect of the accessions in TL variation (ANOVA, $P<0.000$ ) (Suppl. Table 4) and mean values ranged from 10.59 (A-09) to 73.11 (Ag-06), with A-09 being significantly worse than the group of accessions with the highest TL values, a mixture of Capsicum spp. (B-10, B-13, B-14, P-02, Ag01, P-01 and Ag-06) (Table 3). For LA, also the ANOVA detected significant differences $(\mathrm{P}<0.000)$ (Suppl. Table 4). Thus, mean values were comprised between 2.74 (P-01) and 14.18 (Ag-06), although only P-01 was significantly better than Ag-04 and Ag-06 (Table 3). Regarding the LA/TL ratio, mean values ranged from 0.08 (P-02) to 0.62 (A-09) and two groups of accessions differed significantly for this trait, with two $C$. pubescens and a $C$. baccatum (P-02, $\mathrm{P}-01$ and $\mathrm{B}-13$ ) showing LA/TL ratios considerably lower than the two C. annuum accessions A-21 and A-09 (Table 3). Significant differences were also found for MAA, and mean values ranged 0.09 (Co-01) to 0.41 (A-18), although only significant differences were found the 25 best accessions and the worst accession (A-18) (Table 3). Finally, significant differences were also found in CII (ANOVA, $\mathrm{P}<0.000$ ) (Suppl. Table 4), with mean values ranging from $0.12(\mathrm{P}-02)$ to 0.41 (A-18), and the best six accessions (P-02, P-01, Ag-01, Co-01, B-11 and B-13) differed significantly from the two worst accessions (Table 3 ). 


\section{Combined analysis of results}

In order to have a general perspective of the performance of all the accessions evaluated, the z-score mean for CII was calculated for those accessions evaluated in the two trials (Suppl. Tables 5, 6 and 7). By plotting $\mathrm{CII}$ at $30 \mathrm{DAI}$ against $\mathrm{CII}$ at $60 \mathrm{DAI}$ it can be clearly observed that many genotypes had a consistent response against infection through the time. Thus, the CII z-score means of A-06, A-23, Ag-01, Ag-02 and C-05, had a consistent good response against the infection along the 30-60 DAI period (Figure 3, orange quadrant on the left), in contrast to the accession A-21, which showed a consistent bad response (Figure 3, orange quadrant on the right). In comparison, accessions like A-02, A- 04 and A- 08 showed high values of infection at $30 \mathrm{DAI}$, but good response (low CII values) at $60 \mathrm{DAI}$, suggesting a recovery of the plants. Finally accessions plotted at the left-top corner of the graph such as A-10 had worse values at 60 DAI than at $30 \mathrm{DAI}$ (Figure 3, blue quadrant).

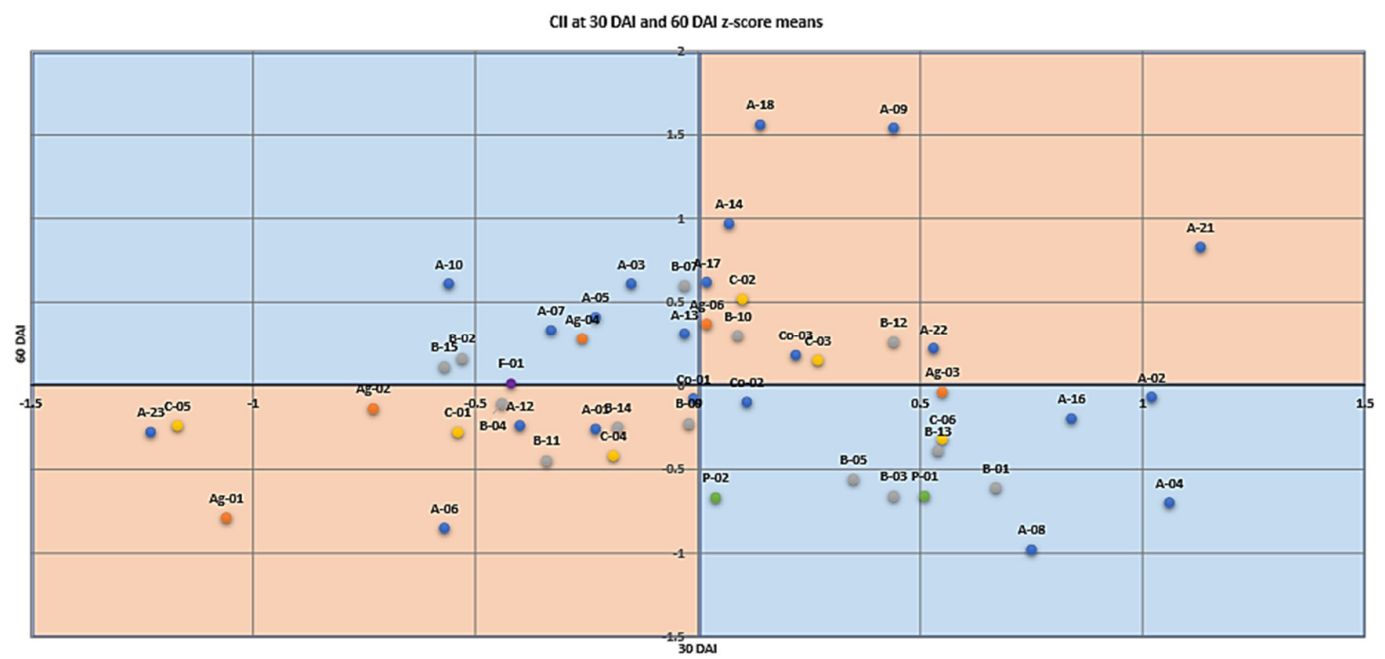

Figure 3. CII at 30 and $60 \mathrm{DAI} z$-score means

Color by spp. Red quadrants are steady responses, down-left as good, up-right as bad response. Blue quadrants are changing responses, up-left getting worst, and down-right getting better

\section{Discussion}

In this work, several Capsicum sp. accessions have been tested for their resistance to PM. Differences in the architecture, size, number of leaves and vigour among accessions could difficult the comparison of their level of tolerance. In addition, dichotomous evaluations for presence and absence of symptoms have been described as inefficient to properly define the resistance to L. taurica (Lin et al., 2019). The use of a composite index mitigated those effects allowing a fair comparison.

Our findings indicate that responses to PM infection were highly dependent on the DAI readings. This agrees with other authors who found differences in the response to $L$. taurica inoculation according to the stage of development (de Souza and Café-Filho, 2003). In this study, plants had the most severe infection at 30 DAI while the responses at $60 \mathrm{DAI}$ were variable among accessions. Some of them showed a remarkable recovery after a period with no inoculum pressure, while others showed similar symptoms and tolerance level at both evaluation times and, even, other accessions showed an increase in the symptoms at 60 DAI.

The resistance to PM is complex as it may be effective at different steps of the infection cycle, being also dependent on the plant age. Daubeze et al. (1995) estimated 2.6-3 genetic factors for seedlings and lighter inoculum pressure, and 5 genetic factors for older and strongest infection pressure. In this regard, leaf shedding is a common mechanism to deal with infection, that allows the plant to survive and sometimes recover. However, the production of photoassimilates is dramatically decreased during a period of time which can be 
limiting for plant development and fruit setting. If defoliation takes place during the fruiting time, fruits can be exposed to excessive irradiation, increasing the risk of sunscald (Elad et al., 2007; Sudha and Lakshmanan, 2009). Therefore, leaf shedding is considered as an indicator of susceptibility.

Other responses to PM infection are more valuable for hampering the development of the fungus, especially those which block the invasion of the host cells by the pathogen from the early stages. This is the action of the recessive genes CaMlo1 and CaMlo2 which are directly involved in cell death and formation of reactive oxygen species, thus avoiding appressorium establishment and germ tube penetration (Kim and Hwang, 2012). Or the classical response of the R-genes, as the PMR1 gene, a major nucleotide-binding site leucine-rich repeat-type gene (NBS-LRR) recently identified (Jo et al., 2017) that detect pathogenic effectors and initiate immune responses. Even so, resistance to PM has a quantitative nature, from which, only some QTLs have been elucidated (Lefevre et al., 2003; Eggink et al., 2016; Gabor et al., 2017).

As expected from the quantitative nature of the resistance to PM, our results showed a continuum of responses to $L$. taurica infection in the different accessions. To record the reaction at two different DAI allowed us to select accessions with a consistent response of low infection over the time, such as Ag-01, Ag-02, A-06, A23, belonging to $C$. annuum and C-05, from $C$. chinense. On the contrary to other authors (Blat et al., 2005 and 2006; McCoy and Bosland, 2019), our findings did not identified sources of resistance from the $C$. baccatum accessions tested here.

The best results of the experiments corresponded to $\mathrm{Ag}-01$ in both trials, and other promising source of variation was Ag-02, although it showed a lower performance in the second trial. Both materials belong to $C$. annuum var. glabriusculum also known as chiltepins. This wild taxon, phylogenetically close to $C$. annumm (Pereira-Dias et al., 2019), is a new source of resistance or tolerance genes only suggested previously by McCoy and Bosland (2019). Due to their cross- compatibility with $C$. annumm they offer the opportunity of easy introgressions of genes of interest by sexual hybridization, even easier than $C$. chinense or $C$. frutescens (Manzur et al., 2015). In addition, the morphology of the leaves with higher hair density in Ag-01 than that observed in other accessions tested may suggest the presence of unexplored physical defense mechanism, as suggested in other species (Kono et al., 2018).

\section{Conclusions}

The resistance to powdery mildew is controlled by several genes and the level of resistance vary with the age of the plant and the genetic background. Although some sources of resistance have been described previously, the search for novel tolerant accessions is still in course. Traditionally, high tolerance to powdery mildew has been found in other species than $C$ annuum. However, here we have found high tolerance within the $C$. annuum taxon, in the var. glabriusculum which may facilitate the introgression of resistance genes into commercial varieties of common peppers. Further studies in adult plants, under several environment conditions and genetic analysis may confirm the potential of accessions of $C$. annuum var. glabriusculum as novel sources of resistance.

\section{Authors' Contributions}

Conceptualization: IIMM, ARB, JJLR and AF; Data curation: IIMM and AF; Formal analysis: IIMM and AF; Funding acquisition: IIMM, ARB and AF; Investigation: IIMM, ARB and AF; Methodology: IIMM, ARB and AF; Project administration: IIMM; Resources: ARB; Visualization: IIMM, MJP, JJLR and AF; Writing - original draft IIMM and AF; Writing - review and editing: IIMM, ARB, MJP, JJLR, ASB and AF.

All authors read and approved the final manuscript. 


\section{Acknowledgements}

IIMM acknowledges his work was supported by CONACYT-CONCYTEP pre-doctoral scholarship number 47274 by the Mexican government. This work has been partially financed by the projects RTA201400041-C02-02 y PID2019-110221RR-C32, from Spain's Plan Nacional, INIA and FEDER/ERDF funds.

\section{Conflict of Interests}

The authors declare that there are no conflicts of interest related to this article.

\section{References}

Aguilar-Meléndez A, Morrell PL, Roose ML, Kim SC (2009). Genetic diversity and structure in semiwild and domesticated chiles (Capsicum annuum; Solanaceae) from Mexico. American Journal of Botany 96(6):11901202. https://doi.org/10.3732/ajb.0800155

Albert R, Künstler A, Lantos F, Adám AL (2017). Graft-transmissible resistance of cherry pepper (Capsicum annuum var. cerasiforme) to powdery mildew (Leveillula taurica) is associated with elevated superoxide accumulation, NADPH oxidase activity and pathogenesis-related gene expression. Acta Physiologiae Plantarum 39:53. https://doi.org/10.1007/s11738-017-2353-5

Bai Y, Huang CC, van der Hulst R, Meijer-Dekens F, Bonnema G, Lindhout P (2003). QTLs for tomato powdery mildew resistance (Oidium lycopersici) in Lycopersicon parviflorum G1.1601 co-localize with two qualitative powdery mildew resistance genes. Molecular Plant-Microbe Interactions 16(2):169-176. https://doi.org/10.1094/MPMI.2003.16.2.169

Blat SF, da Costa CP, Vencovsky R, Sala FC (2005). Inheritance of reaction to Leveillula taurica (Lev.) Arn. in Capsicum annuum L. Scientia Agricola 62(1):40-44. https://doi.org/10.1590/S0103-90162005000100008

Blat SF, da Costa CP, Vencovsky R, Sala FC (2006). Hot pepper (Capsicum chinense, Jacq.) inheritance of reaction to powdery mildew. Scientia Agricola 63(5):471-474. https://doi.org/10.1590/S0103-90162006000500008

Daubeze AM, Hennart JW, Palloix A (1995). Resistance to Leveillula taurica in pepper (Capsicum annuum) is oligogenically controlled and stable in Mediterranean regions. Plant Breeding 114:327-332. https://doi.org/10.1111/j.1439-0523.1995.tb01243.x

de Souza VL, Café-Filho AC (2003). Resistance to Leveillula taurica in the genus Capsicum. Plant Pathology 52:613-619. https://doi.org/10.1046/j.1365-3059.2003.00920.x

Eggink PM, D'hoop BB, Brouwer M, and Deniau AX (2016). Resistance against Leveillula taurica in Pepper. U.S. Patent No 9, 351, 451. Washington, DC: U.S. Patent and Trademark Office.

Elad Y, Messika Y, Brand M, David DR, Sztejnberg A (2007). Effect of microclimate on Leveillula taurica powdery mildew of sweet pepper. Phytopathology 97(7):813-824. https://doi.org/10.1094/PHYTO-97-7-0813

FAOSTAT (2021). Statistic division, Food and Agriculture Organization of the United Nations. Rome, Italy. Retrieved 2021 March 01 from http://www.fao.org/faostat/

Gabor BK, Just BJ, Huang C, Jones CM, Vreugdenhil D, Kniskern JM, ... Xiang W (2017). Methods and compositions for producing Capsicum plants with powdery mildew resistance. U.S. Patent No 9,689,045. Washington, DC: U.S. Patent and Trademark Office.

Hayano-Kanashiro C, Gámez-Meza N, Medina-Juárez LÁ (2016). Wild pepper Capsicum annuum L. var. glabriusculum: Taxonomy, plant morphology, distribution, genetic diversity, genome sequencing, and phytochemical compounds. Crop Science 56(1):1-11. https://doi.org/10.2135/cropsci2014.11.0789

Hoshmand R (2020). Design of experiments for agriculture and the natural sciences ( $2^{\text {nd }}$ edition). Chapman and Hall/CRC.

Jo J, Venkatesh J, Han K, Lee HY, Choi GJ, Lee HJ, Choi D, Kang BC (2017). Molecular mapping of PMR1, a novel locus conferring resistance to powdery mildew in pepper (Capsicum annuum). Frontiers in Plant Science 8: 2090. https://doi.org/10.3389/fpls.2017.02090 
Kim DS, Hwang BK (2012). The pepper MLO gene, CaMlo2, is involved in susceptibility cell death response and bacterial and oomycete proliferation. The Plant Journal 72:843-855. https://doi.org/10.1111/tpj.12003

Kono A, Ban Y, Mitani N, Fujii H, Sato S, Suzaki K, ... Sato A (2018). Development of SSR markers linked to QTL reducing leaf hair density and grapevine downy mildew resistance in Vitis vinifera. Molecular Breeding 38:138. https://doi.org/10.1007/s11032-018-0889-8

Kraft KH, Luna-Ruíz J, Gepts P (2013). A new collection of wild populations of Capsicum in Mexico and the southern United States. Genetic Resources and Crop Evolution 60(1):225-232. https://link.springer.com/article/10.1007/s10722-012-9827-5

Lee OH, Hwang HS, Kim JY, Han JH, Yoo YS, Kim BS (2001). A search for sources of resistance to powdery mildew (Leveillula taurica (Lév.) Arn) in pepper (Capsicum spp.). Korean Journal of Horticultural Science and Technology 19(1):7-11.

Lefebvre V, Daubèze AM, Rouppe van der Voort J, Peleman J, Bardin M, Palloix A (2003). QTLs for resistance to powdery mildew in pepper under natural and artificial infections. Theoretical and Applied Genetics 107:661-666.

Lin K, Gong L, Huang Y, Liu C, Pan J (2019). Deep learning-based segmentation and quantification of cucumber powdery mildew using convolutional neural network. Frontiers in Plant Sciences 10:155. https://doi.org/10.3389/fpls.2019.00155

Luna-Ruiz JdJ, Pérez-Chávez MS, Martínez-de-Anda JA, Sosa-Ramírez J (2018). Distribución ecogeográfica del chile silvestre en México y su conservación ex situ. In: Aguilar-Meléndez A, Vásquez-Dávila MA, Katz E, HernándezColorado MR (Eds). Los chiles que le dan sabor al mundo. IRD Éditions. https://doi.org/10.4000/books.irdeditions.30934

Manzur JP, Fita A, Prohens J, Rodríguez-Burruezo A (2015). Successful wide hybridization and introgression breeding in a diverse set of common peppers (Capsicum annuum) using different cultivated ají (C. baccatum) accessions as donor parents. PLoS One 10:12. https://doi.org/10.1371/journal.pone.0144142

McCoy JE, Bosland PW (2019). Identification of resistance to powdery mildew in Chile pepper. HortScience 54(1):4-7. https://doi.org/10.21273/HORTSC113596-18

Molot PM, Leroux JP, Diop-Bruckler M (1990). Leveillula taurica (Lév) Arn: cultures axéniques, biologie et spécificité parasitaire [Leveillula taruica (Lév) Arn: axenic cultures, biology and parasite specificity]. Agronomie 10(7):551559. https://hal.archives-ouvertes.fr/hal-00885316

Molot PM, Leroux JP, Ferriere H (1987). Les oÏdiums des cucurbitacées. II. Mise au point d'une technique de conservation des souches en culture axénique [Powdery mildew of cucurbits. II. Development of a technique for the conservation of strains in axenic culture]. Agronomie 7(5):339-343. https://hal.archives-ouvertes.fr/hal00884999

Özer N, Kün A, İlbi H (2018). Detached leaf test for evaluation of resistance to powdery mildew in pepper. Agricultural Research \& Technology 14:3. https://doi.org/10.19080/ARTOAJ.2018.14.555923

Parisi M, Alioto D, Tripodi P (2020). Overview of biotic stresses in pepper (Capsicum spp.): Sources of genetic resistance, molecular breeding and genomics. International Journal of Molecular Sciences 21(7):2587. https://doi.org/10.3390/ijms21072587

Pereira-Dias L, Vilanova S, Fita A, Prohens J, Rodríguez-Burruezo A (2019). Genetic diversity, population structure, and relationships in a collection of pepper (Capsicum spp.) landraces from the Spanish center of diversity revealed by genotyping-by-sequencing. Horticulture Research 6:54. https://doi.org/10.1038/s41438-019-0132-8

Robbins MGL, Hoffmann DRT, Wang FV (2017). Anticarcinogenic flavonoids in different fruits commonly consumed. Plant Sciences Journal 40:2379-2383.

Sudha A, Lakshmanan P (2009). Integrated disease management of powdery mildew (Leveillula taurica (Lev.) Arn.) of chilli (Capsicum annuum L.). Archives of Phytopathology and Plant Protection 42:299-317. https://doi.org/10.1080/03235400601037198

Zheng Z (2012). Exploration of MLO-based resistance in vegetable crops. PhD Thesis, Wageningen University, Netherlands.

Zheng Z, Nonomura T, Bóka K, Matsuda Y, Visser RGF, Toyoda H, ... Bai Y (2013). Detection and quantification of Leveillula tauricagrowth in pepper leaves. Phytopathology 103:623-632. https://doi.org/10.1094/PHYTO-08$12-0198-R$ 
OPEN ACCESS

(c) (i)

The journal offers free, immediate, and unrestricted access to peer-reviewed research and scholarly work. Users are allowed to read, download, copy, distribute, print, search, or link to the full texts of the articles, or use them for any other lawful purpose, without asking prior permission from the publisher or the author.

License - Articles published in Notulae Botanicae Horti Agrobotanici Cluj-Napoca are Open-Access, distributed under the terms and conditions of the Creative Commons Attribution (CC BY 4.0) License. (C) Articles by the authors; UASVM, Cluj-Napoca, Romania. The journal allows the author(s) to hold the copyright/to retain publishing rights without restriction. 